\title{
Expected Monetary Impact of Oncotype DX Score-Concordant Systemic Breast Cancer Therapy Based on the TAILORx Trial
}

\author{
Angela Mariotto, Jinani Jayasekerea, Valentina Petkov, Clyde B. Schechter, \\ Lindsey Enewold, Kathy J. Helzlsouer, Eric J. Feuer, Jeanne S. Mandelblatt
}

See the Notes section for the full list of authors' affiliations.

Correspondence to: Jeanne Mandelblatt, MD, MPH, Georgetown-Lombardi Comprehensive Cancer Center, Cancer Prevention and Control Program, 3300 Whitehaven Street, Suite 4100, Washington, DC 20007 (mandelbj@georgetown.edu).

\begin{abstract}
Background: TAILORx demonstrated that women with node-negative, hormone receptor-positive, HER2-negative breast cancers and Oncotype DX recurrence scores (RS) of 0-25 had similar 9-year outcomes with endocrine vs chemo-endocrine therapy; evidence for women aged 50 years and younger and RS 16-25 was less clear. We estimated how expected changes in practice following the trial might affect US costs in the initial 12 months of care (initial costs).

Methods: Data from Surveillance, Epidemiology, and End Results (SEER), SEER-Medicare, and SEER-Genomic Health Inc datasets were used to estimate Oncotype DX testing and chemotherapy rates and mean initial costs pre- and post-TAILORx (in 2018 dollars), assuming all women received Oncotype DX testing and score-suggested therapy posttrial. Sensitivity analyses tested the impact on costs of assumptions about compliance with testing and score-suggested treatment and estimation methods.

Results: Pretrial mean initial costs were $\$ 2.816$ billion. Posttrial, Oncotype DX testing costs were projected to increase from $\$ 115$ to $\$ 231$ million and chemotherapy use to decrease from $25 \%$ to $17 \%$, resulting in initial care costs of $\$ 2.766$ billion, or a net savings of $\$ 49$ million (1.8\% decrease). A small net savings was seen under most assumptions. The one exception was if all women aged 50 years and younger with tumors with RS 16-25 elected to receive chemotherapy, initial care costs could increase by $\$ 105$ million (4\% increase).

Conclusions: Personalizing breast cancer treatment based on tumor genetic profiles could result in small cost decreases in the initial 12 months of care. Studies are needed to evaluate the long-term costs and nonmonetary benefits of personalized cancer care.
\end{abstract}

Breast cancer treatment recommendations are increasingly personalized based on tumor genomic profile data. The landmark TAILORx trial assessed the benefits of chemotherapy by specific categories of genomic profile test scores among women diagnosed with early-stage, lymphnode-negative, hormone receptor-positive, HER2-negative breast cancer (1). Women with tumors having Oncotype DX (Genomic Health Inc, Redwood City, CA) recurrence scores (RS) of 11-25 had similar distant recurrence rates and breast cancer deaths with endocrine vs chemo-endocrine treatment (1), suggesting that chemotherapy could be omitted in this group. Results also strengthened the support for omission of chemotherapy among women with cancers with RS 0-10 (1-3).

The practice implications of the TAILORx trial are potentially far-reaching for cancer care costs and quality of life, because nearly $60 \%$ of US women diagnosed annually have breast cancers of the types included in the trial (4). Although tumor genomic profile testing was recommended by professional organizations $(5,6)$ prior to publication of the TAILORx results, rates of testing in the United States only ranged from $25 \%$ to $50 \%$ of eligible women $(7,8)$ and was selected based on patient characteristics and perceived clinical utility (9). The evidence 
provided by the TAILORx trial is likely to increase use of gene expression profile testing, leading to potential cost savings (and harm reduction) by omitting chemotherapy among women who will not benefit from it.

We used population-based data and Medicare costs to project the societal economic impact of practice changes based on the TAILORx trial results. We compared pre- and posttrial costs in the first 12 months following diagnosis (initial costs) among women who would have been eligible for TAILORx, assuming that posttrial all women would receive Oncotype DX testing and follow score-suggested therapy. The results are intended to inform policy and payer discussions about the costs of personalized cancer care in the United States.

\section{Methods}

We conducted new analyses of several sources of de-identified US population-based data to estimate the monetary impact of changes in practice post-TAILORx. The study was considered exempt by the Georgetown University Institutional Review Board and the National Institutes of Health's Office of Human Subjects Research Review Board.

\section{Population}

We estimated incidence rates and numbers of women with TAILORx-eligible breast cancers in the pretrial (2010-2015) and posttrial (2018) periods. First, we calculated average incidence rates from 2010 to 2015 for women who were ages 18-75 years and had node-negative, estrogen-, and/or progesteronereceptor-positive or borderline (hormone receptor [HR]-positive), and HER2 (or unknown) tumors with sizes of $1.1-5.0 \mathrm{~cm}$ or $0.5-1.0 \mathrm{~cm}$ and intermediate or high grade (10). Because of the 5year age groupings in the Surveillance, Epidemiology, and End Results (SEER) data, we included women ages 15-74 years (vs 18-74 in TAILORx). We excluded women diagnosed at autopsy or death certificate. We then applied the resulting age- (and race-) specific incidence rates to the US female population (11) to estimate the population of women with breast cancer diagnosed in the United States in 2018 whose care might be affected by the TAILORx results ( $\mathrm{n}=67563$ women).

\section{Oncotype DX Testing}

We focused on the Oncotype DX test because it was used in the TAILORx trial (1) and has also been linked to population data. We used linked SEER-Genomic Health Inc data (12) from women diagnosed with TAILORx-eligible breast cancers and known Oncotype DX RS to estimate the age-specific rates of testing in the pretrial period (Supplementary Table 1, available online). Posttrial, we assumed $100 \%$ use of Oncotype DX testing.

\section{Recurrence Score (RS) Distribution}

The proportion of women with breast cancers in each Oncotype DX RS category $(0-10,11-15,16-25,26-31$, and >31) was based on the sum of the observed proportions in the linked SEERGenomic Health Inc data (12) from women diagnosed with TAILORX-eligible breast cancers combined with imputed scores for untested women. We imputed RS using a categorical logistic regression model conditional on age and clinical-pathological characteristics (Supplementary Table 2, available online).

\section{Chemotherapy}

Chemotherapy is often slightly underreported in SEER (13). Therefore, we used a combination of SEER and SEER-Medicare data (14) to estimate true chemotherapy rates pre-TAILORx. Chemotherapy rates were determined based on 2010-2015 SEER-Medicare data for trial-eligible women ages 65-74 years who were continuously enrolled in Medicare fee-for-service Parts A and B during the first 12 months postcancer diagnosis. A comparison of SEER and SEER-Medicare chemotherapy rates in this group showed SEER rates to be $14 \%$ lower than Medicare rates. Thus, to calculate chemotherapy rates before TAILORx among persons younger than 65 years, we applied a 14\% inflation factor to the SEER rates (Supplementary Table 3, available online); alternative rates were tested in sensitivity analyses. Posttrial, we assumed that $100 \%$ of women received scoresuggested therapy based on TAILORx results (ie, 0-25 no chemotherapy; $\geq 26$ chemotherapy).

\section{Initial Care Costs}

We used previously developed methods to estimate pretrial initial costs for trial-eligible women ages 65-74 years (and continuously enrolled in Medicare fee-for-service Parts A and B) using linked 2010-2015 SEER-Medicare data $(15,16)$. These methods compared mean per person costs of women with breast cancer to matched noncancer control subjects. Costs included Medicare reimbursements, copayments, co-insurance, and deductible amounts by age group and receipt of chemotherapy, where chemotherapy costs included treatment and toxicity management costs (12). We assumed that chemotherapy costs were similar in the pre- and posttrial periods, because there have not been major regimen changes in the study period. We did not include the costs of hormonal therapy, because all women in TAILORx received hormonal therapy.

Because women younger than 65 years generally receive more aggressive systemic therapy, we multiplied the mean Medicare costs per woman by a factor of 1.3 for women younger than 65 years (17); alternative inflation rates were examined in sensitivity analyses.

Initial care costs attributable to Oncotype DX testing were estimated as the proportion receiving testing multiplied by the Medicare reimbursement rate of $\$ 3416$ per test (18). We added Oncotype DX testing costs separately to costs in the pre- and posttrial periods based on their rates of use to separate effects of the TAILORx results on testing vs chemotherapy costs.

To estimate total US initial care costs, we multiplied the mean initial costs per woman with a TAILORx-eligible breast cancer by the number of women in the analytic population based on age, chemotherapy, and test use. All costs were calculated in 2018 dollars based on the Medical Care Component of the CPI (19).

\section{Analysis}

We compared the total initial care costs associated with testing and chemotherapy use in the period before and after the trial. Costs before the trial were based on the patterns of care observed from 2010 to 2015 . After the trial, cost calculations assumed that $100 \%$ of women would have Oncotype DX testing and that all tested women would adhere to treatment prescribed by the trial (ie, endocrine therapy if RS 0-25 and chemoendocrine therapy if $\mathrm{RS} \geq 26$ ). 
One-way sensitivity analyses were used to test the impact on cost results of several alternative assumptions about changes in practice patterns (Supplementary Table 4, available online). First, because the trial suggested some benefit of chemotherapy in women aged 50 years and younger with RS 1625 , we tested the impact of assuming that all of these younger women would receive chemotherapy. Second, because not all women choose to have score-suggested therapy $(7,20)$, we assumed that the proportion of women with RS 26-30 and/or $\geq 31$ who received chemotherapy would be similar to the pretrial age-specific proportions among those with $\mathrm{RS} \geq 31$. We also assumed that some women with RS 0-25 would still choose chemotherapy at a rate similar to women with RS 0-10 in the pretrial era. Third, because the true distribution of RS across the entire eligible female breast cancer population was not known, we tested the impact of the imputation accuracy by varying the predicted probability of being in each score category using the lower and upper confidence intervals, adjusting the other groups proportionally in the opposite direction.

All one-way sensitivity analyses were then repeated separately while varying the previously described correction factors for the estimation of chemotherapy rates ( $7 \%$ and $21 \%$ vs $14 \%$ ) and costs (1.2 and 1.4 vs 1.3) among women 64 years or younger (Supplementary Tables 5 and 6, available online).

\section{Results}

\section{Pretrial}

In the pretrial era, the overall Oncotype testing rate among women with HR-positive, HER early-stage breast cancers was $49.8 \%$, but varied by age from $34.8 \%$ to $57.2 \%$ (Table 1 ). Among those tested, most women had tumors with RS of 11-25. Further, chemotherapy use varied by RS category and age. Despite chemotherapy being recommended in the pre-TAILORx period for women with RS $\geq 31$, more than $10 \%$ of these women did not receive chemotherapy; similarly, a small percent of women with RS 0-10 received chemotherapy, although it was not recommended.

The mean initial costs in the pre-TAILORx period (excluding Oncotype testing) were estimated to range across age groups from \$52 100 to $\$ 67800$ per woman for those who received chemotherapy vs $\$ 26600$ to $\$ 34600$ for those not receiving chemotherapy (Table 2). In the pretrial period, total expenditures during the initial care phase were estimated to be $\$ 2.701$ billion (Table 3). The cost of Oncotype DX testing during this period (ie, testing among about $50 \%$ of women) was estimated to be $\$ 115$ million, for a total cost of $\$ 2.816$ billion.

\section{Post-TAILORx Trial}

After the TAILORx trial, chemotherapy use was projected to decrease from $25 \%$ to $17 \%$, saving $\$ 338$ million in initial chemotherapy costs per year (Table 3). Universal Oncotype DX testing would increase testing costs from \$115 to \$231 million, for an estimated total initial care cost of $\$ 2.766$ billion in the posttrial period. Compared to the pretrial period, this would represent a net savings of $\$ 49$ million, or $1.8 \%$ of the pretrial total initial care costs.

\section{Sensitivity Analyses}

The predicted small savings in total initial care costs if care followed the TAILORx trial results was robust across most assumptions and ranged from savings of $\$ 32$ to $\$ 102$ million (Figure 1). However, if $100 \%$ of women aged 50 years and younger who had tumors with RS 16-25 received chemotherapy, total initial care costs would increase by $\$ 105$ million from costs in the pretrial period (a $4 \%$ increase in total costs). If chemotherapy use among women with RS $\geq 26$ was not universal, but remained similar to the pre-TAILORx era for women with scores $\geq 31$, the cost saving could increase to more than $\$ 102$ million.

Savings in total initial care costs based on different assumptions about the distribution of scores across categories or adjustment to estimate chemotherapy use and medical costs for women with breast cancer ages 15-64 years did not change the results substantially (Table 4). The smallest and largest changes in costs were estimated to be an increase of \$132 million and a savings of $\$ 117$ million in total initial care costs.

\section{Discussion}

This study shows that changes in practice from current patterns to new standards expected based on the TAILORx trial have the potential to affect the costs of total initial cancer care for women diagnosed with node-negative, HR-positive, HER2negative breast cancer. If treatment follows trial-suggested care, there is likely to be a small net cost decrease (\$49 million) during the initial 12 months of breast cancer care, despite an increase in the cost of testing. This projection was robust to a range of assumptions. However, if all women aged 50 years and younger with RS 16-25 received chemotherapy, there could be a small net increase in initial costs.

Our results suggest that personalizing care by selecting chemotherapy based on RS has the potential to lower short-term costs, even after considering the added costs of expanded Oncotype DX testing. The net savings are projected to be $1.8 \%$ of the pretrial total initial breast cancer care costs and are a consequence of lower chemotherapy rates than current patterns.

Economic results are only a partial picture of the total benefits to women from changes in care based on evidence from the TAILORx trial. There are important nonmonetary benefits of the expected new treatment paradigm, including targeting of chemotherapy to those most likely to benefit and avoiding harms among those with little to no potential benefit in terms of reduced recurrence risks. Compared to endocrine treatment, chemo-endocrine therapy may lead to more extensive shortand long-term morbidity, including fatigue, hair loss, heart problems, neurotoxicity, early menopause, and greater personal disruption from cancer treatment. Knowledge of RS may also potentially decrease (or increase) worry about future recurrence $(21,22)$ and affect confidence in treatment decisions (23).

The only situation where personalizing care based on Oncotype DX results could result in a net increase in initial care costs was if women aged 50 and younger with tumors with RS of 16-25 all chose to receive chemotherapy, because this group was previously considered as having an intermediate risk of recurrence and did not uniformly use chemotherapy. The absolute reduction in risk of recurrence with chemotherapy seen in TAILORx was small for this subgroup (1); therefore, it is not clear what the actual chemotherapy patterns will be for this subgroup moving forward.

There are several caveats that should be considered in interpreting our results. First, to project the possible savings because of personalized care, our analysis assumed that Oncotype DX testing would be universal posttrial and that women would receive score-suggested therapy. However, there are many other 
Table 1. Rates of Oncotype DX testing, recurrence score distributions, and chemotherapy use by age group and time period among women with early-stage, hormone receptor-positive, HER2-negative breast cancer

\begin{tabular}{|c|c|c|c|c|c|c|c|c|}
\hline \multirow[b]{2}{*}{ Variable } & \multicolumn{4}{|c|}{ Pretrial $^{*}, \dagger$} & \multicolumn{4}{|c|}{ Posttrial $\neq$} \\
\hline & $\begin{array}{l}\text { Age } 15-49 \\
\text { years }\end{array}$ & $\begin{array}{l}\text { Age } 50-64 \\
\text { years }\end{array}$ & $\begin{array}{l}\text { Age } 65-69 \\
\text { years }\end{array}$ & $\begin{array}{c}\text { Age } 70-74 \\
\text { years }\end{array}$ & $\begin{array}{l}\text { Age } 15-49 \\
\text { years }\end{array}$ & $\begin{array}{l}\text { Age } 50-64 \\
\text { years }\end{array}$ & $\begin{array}{l}\text { Age 65-69 } \\
\text { years }\end{array}$ & $\begin{array}{c}\text { Age } 70-74 \\
\text { years }\end{array}$ \\
\hline Total number of women & 19332 & 43859 & 18351 & 14987 & \multicolumn{4}{|c|}{$N=67563$} \\
\hline Women tested, \% & 57.2 & 55.3 & 46.3 & 34.8 & 100 & 100 & 100 & 100 \\
\hline \multicolumn{9}{|c|}{ Oncotype DX RS distribution, \% } \\
\hline $0-10$ & 17.1 & 21.0 & 25.4 & 27.3 & 14.2 & 19.9 & 25.4 & 27.9 \\
\hline $11-15$ & 25.5 & 25.1 & 23.8 & 24.6 & 23.2 & 23.8 & 23.7 & 24.9 \\
\hline $16-25$ & 41.2 & 38.6 & 35.6 & 34.0 & 40.9 & 38.2 & 35.4 & 33.9 \\
\hline $26-30$ & 7.1 & 7.6 & 7.6 & 7.0 & 9.6 & 8.4 & 7.5 & 6.6 \\
\hline $31-100$ & 7.0 & 7.6 & 7.7 & 7.1 & 12.2 & 9.7 & 8.0 & 6.7 \\
\hline \multicolumn{9}{|c|}{ Chemotherapy rates by Oncotype DX RS, \% } \\
\hline $0-10$ & 4.7 & 1.5 & 3.7 & 3.7 & 0 & 0 & 0 & 0 \\
\hline $11-15$ & 7.0 & 3.2 & 4.3 & 4.9 & 0 & 0 & 0 & 0 \\
\hline $16-25$ & 36.2 & 23.4 & 16.9 & 12.0 & 0 & 0 & 0 & 0 \\
\hline $26-30$ & 79.8 & 69.1 & 46.1 & 40.2 & 100 & 100 & 100 & 100 \\
\hline $31-100$ & 90.9 & 89.9 & 77.2 & 69.8 & 100 & 100 & 100 & 100 \\
\hline Untested & 63.4 & 32.5 & 14.7 & 10.8 & - & - & - & - \\
\hline
\end{tabular}

*Pre-TAILORx Oncotype DX testing and score distribution results were estimated using SEER data linked to Oncotype DX data from Genomic Health Inc for women diagnosed at ages 15-74 years in 2010-2015 who met the TAILORx inclusion criteria ( $\mathrm{N}=96529)$. TAILORx trial inclusion criteria included women diagnosed at ages $18-$ 74 years, node-negative, estrogen, and/or progesterone receptor positive or borderline, HER2-negative, and tumor sizes $1.1-5.0 \mathrm{~cm}$ or $0.5-1.0 \mathrm{~cm}$ and intermediate or high grade. Because of the 5-year age grouping in SEER, in this study we included women ages 15-74 years. RS = recurrence scores; SEER = Surveillance, Epidemiology, and End Results.

†Pre-TAILORx chemotherapy use in the first 12 months after cancer diagnosis was estimated using SEER and SEER-Medicare data (see Supplementary Table 3, available online for details).

$\ddagger$ Post-TAILORx, we assumed $100 \%$ Oncotype DX testing and combined observed with predicted score distributions from the pretrial cohort using categorical logistic regression estimates for untested women (see Supplementary Table 2, available online). Chemotherapy rates assumed compliance with TAILORx results.

Table 2. Estimated mean initial costs by chemotherapy use and age for women with early-stage, hormone receptor-positive, HER2-negative breast cancer*

Average costs (per woman) in the initial 12 months of care (2018 US\$)†

\begin{tabular}{|c|c|c|c|}
\hline \multirow[b]{2}{*}{ Therapy } & \multirow[b]{2}{*}{ Age, $y$} & \multicolumn{2}{|c|}{ Average costs (per woman) in the initial 12 months of care (2018 US\$)† } \\
\hline & & $\begin{array}{c}\text { Total costs } \\
\text { Mean }(95 \% \mathrm{CI})\end{array}$ & $\begin{array}{l}\text { Total excluding Oncotype DX test costs } \neq \\
\text { Mean }(95 \% \mathrm{CI})\end{array}$ \\
\hline Chemotherapy & $15-64$ & $\$ 69300$ (64 400 to 74200$)$ & $\$ 67800$ (62 900 to 72700$)$ \\
\hline No chemotherapy & $15-64 \dagger$ & $\$ 36800$ (35 600 to 38000$)$ & $\$ 34600$ (33 400 to 35800$)$ \\
\hline Chemotherapy & $65-69$ & $\$ 61200$ (58 700 to 63700 & $\$ 59100$ (56 700 to 61600$)$ \\
\hline No chemotherapy & $65-69$ & $\$ 31600$ (30 600 to 32600$)$ & $\$ 30100$ (29 100 to 31100$)$ \\
\hline Chemotherapy & $70-74$ & $\$ 53300$ (49 500 to 5700$)$ & $\$ 52100$ (48 400 to 55900$)$ \\
\hline No chemotherapy & $70-74$ & $\$ 28300$ (27 400 to 29200$)$ & $\$ 26600$ (25 700 to 27600$)$ \\
\hline
\end{tabular}

${ }^{*}$ Costs for women ages 65-74 years were based on new analyses of SEER-Medicare data for the subset of women who were continuously enrolled in Medicare fee-forservice Parts A and B during the first 12 months post-cancer diagnosis and who were diagnosed with tumors that match eligibility for the TAILORx trial (node-negative, hormone receptor-positive, HER2-negative breast cancers, sizes $1.1-5.0 \mathrm{~cm})$. CI = confidence interval.

†Costs for women ages 15-64 years were estimated to be 1.3 higher compared to those of women ages 70-74 years (17).

$\ddagger$ Per woman cost of Oncotype DX testing was based on the current Medicare reimbursement rate $\$ 3416$. Cost excluding test $=$ mean initial costs - proportion tested times $\$ 3416$.

factors that affect diagnostic testing and treatment decisions, such as women's preferences, worry about recurrence, and health, and oncologists' assessment of the utility of genomic information, and clinical pathological tumor features (24-26). Other aspects of womens' situations may also drive decisions (27). For instance, a woman with RS 26-30 who is unable to travel for multiple cycles of chemotherapy might opt to not use chemotherapy, especially if she has other tumor features like grade suggesting a good prognosis.

Second, we did not consider how the alignment of payer and provider incentives could affect the actual costs of personalized care. Our results suggest that there are incentives to payers to promote use of Oncotype DX testing, because there are likely to be small short-term cost savings from lower use of chemotherapy. However, for oncologists and infusion centers, there are fewer incentives to decrease rates of chemotherapy administration. It will be important to evaluate how incentive and valuebased reimbursement models that consider de-escalation of treatment will affect actuals patterns of care and costs (28-30).

Third, we only considered initial care costs and not downstream costs associated with distant recurrence among those who omitted chemotherapy but were destined to have recurrent disease. The TAILORx trial provided evidence about the value of personalizing chemotherapy based on Oncotype DX scores as a predictor of the probability of recurrence. Although the trial cut points were selected based on the best knowledge about scores 
Table 3. Total initial care costs pretrial vs potential costs expected in the posttrial period*

\begin{tabular}{|c|c|c|c|c|}
\hline \multirow[b]{2}{*}{ Variable } & \multicolumn{2}{|c|}{ Pretrial† } & \multicolumn{2}{|c|}{ Posttrial $\neq$} \\
\hline & No. of women (\%) & Costs, millions (US\$) & No. of women (\%) & Costs, millions (US\$) \\
\hline Receive chemotherapy & $16620(24.6)$ & $\$ 1.083$ & $11671(16.3)$ & $\$ 745$ \\
\hline No chemotherapy & $50943(75.4)$ & $\$ 1.618$ & $55892(82.7)$ & $\$ 1.791$ \\
\hline Treatment total & $67563(100)$ & $\$ 2.701$ & $67563(100)$ & $\$ 2.536$ \\
\hline Oncotype DX testing & 33634 (49.8) & $\$ 115$ & $67563(100)$ & $\$ 231$ \\
\hline Total costs & - & $\$ 2.816$ & - & $\$ 2.766$ \\
\hline Savings in costs & - & & - & $\$ 49$ \\
\hline
\end{tabular}

*Among women ages 15-74 years with node-negative, hormone receptor-positive, HER2-negative, and sizes 1.1-5.0 cm breast cancers eligible for TAILORx (trial). †Pretrial data were based on observed rates of use of Oncotype DX testing in Surveillance, Epidemiology, and End Results (SEER) data linked to Oncotype DX data from Genomic Health Inc for women diagnosed in 2010-2015. Chemotherapy use was estimated using SEER and SEER-Medicare data.

¥Posttrial assumed $100 \%$ use of Oncotype testing and combined observed with predicted score distributions from the pretrial cohort using categorical logistic regression estimates for untested women (see Supplementary Table 2, available online). Chemotherapy rates assumed compliance with trial results (ie, $0 \%$ chemotherapy among women whose tumors had an Oncotype DX recurrence score of 0-25, and 100\% chemotherapy with scores of $\geq 26$ ).

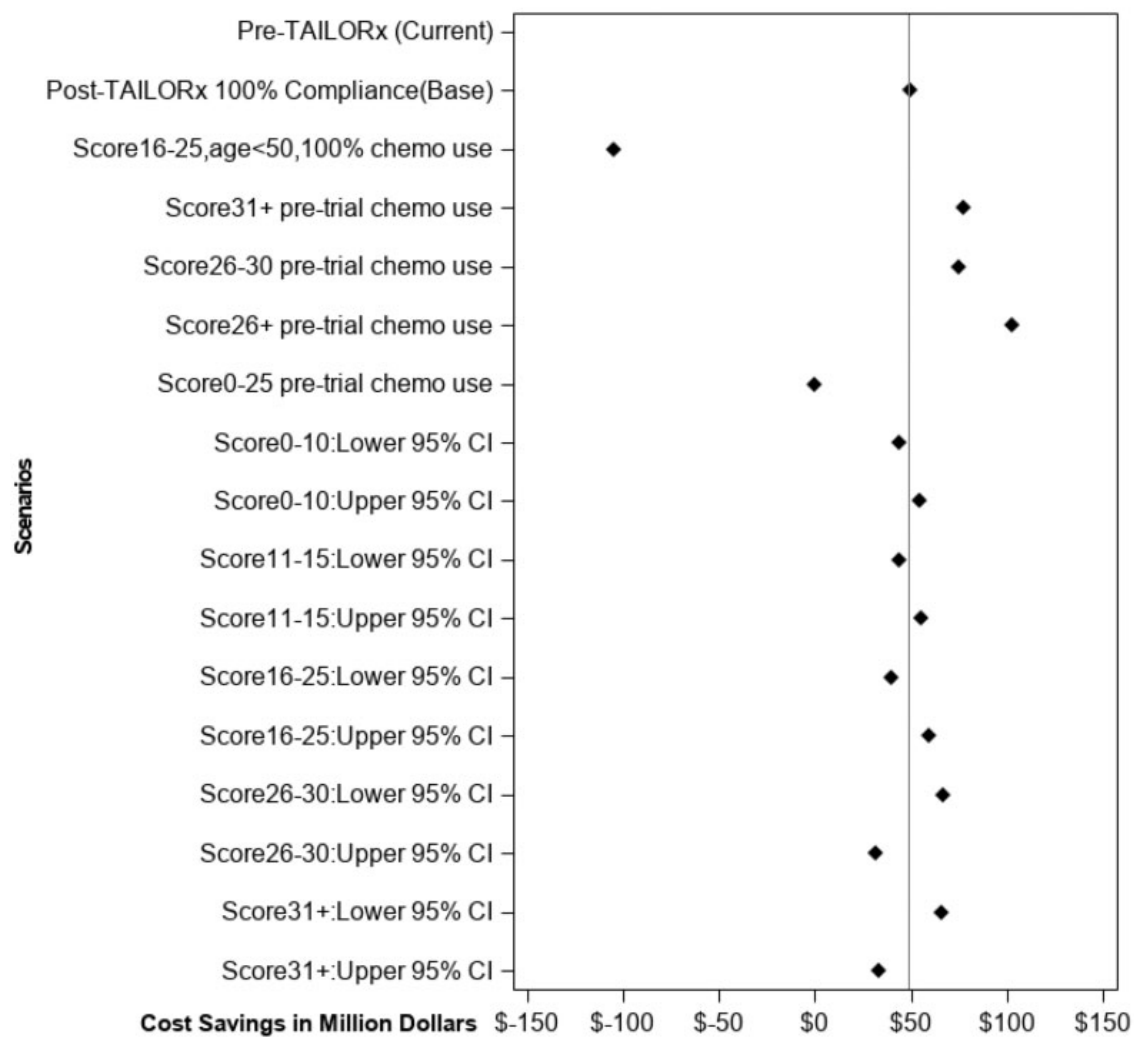

$\begin{array}{rcr}\begin{array}{c}\text { Savings } \\ \text { (Million } \\ \text { US\$) }\end{array} & \begin{array}{c}\text { Total } \\ \text { Costs } \\ \text { Million } \\ \text { US\$) }\end{array} & \begin{array}{c}\text { Women } \\ \text { Receiving } \\ \text { Chemo in } \\ \text { Percent }\end{array} \\ \$ 2,816 & 25 \% \\ \$ 49 & \$ 2,766 & 17 \% \\ \$-105 & \$ 2,920 & 24 \% \\ \$ 77 & \$ 2,738 & 16 \% \\ \$ 75 & \$ 2,741 & 16 \% \\ \$ 102 & \$ 2,713 & 15 \% \\ \$-0 & \$ 2,816 & 20 \% \\ \$ 44 & \$ 2,771 & 18 \% \\ \$ 55 & \$ 2,761 & 17 \% \\ \$ 44 & \$ 2,772 & 18 \% \\ \$ 55 & \$ 2,760 & 17 \% \\ \$ 40 & \$ 2,776 & 18 \% \\ \$ 59 & \$ 2,757 & 17 \% \\ \$ 67 & \$ 2,749 & 16 \% \\ \$ 32 & \$ 2,784 & 18 \% \\ \$ 66 & \$ 2,750 & 16 \% \\ \$ 33 & \$ 2,783 & 18 \% \\ & & \end{array}$

Figure 1. Effects of alternative assumptions on the US total initial care costs pretrial vs potential costs based on patterns of practice expected posttrial. This diagram illustrates the changes in the net costs of initial care of women with node-negative, hormone receptor-positive, HER2-negative, size 1.1-5.0 cm breast cancers from the pre-TAILORx trial (pretrial) to the post-TAILORx trial (posttrial) under differing scenarios based on various parameter values and alternative assumptions. Initial care costs are the total cancer costs in the first 12 months after diagnosis. The solid vertical line represents the base analysis savings comparing the initial care costs prior to the TAILORx trial to the initial care costs post-TAILORx trial. The diamond shapes indicate the savings when the one individual value used in the post-TAILORx scenario analysis was varied. If the diamond shape is to the right of the base case line, it indicates that the alternative value or assumption results in greater cost savings than the base case, whereas diamonds to the left indicate that the alternative value or assumption had less savings or increased costs compared to the base case costs. The scenarios include the following: 1) Subgroups: Chemotherapy use was varied from zero among women with tumors with recurrence scores of 16-25 to 100\% for women in this score category who are aged 50 years or younger. 2) Adherence to score-suggested treatment: These analyses assume that, rather than $100 \%$ of women with scores of $\geq 26$ and/or $\geq 31$ receiving chemotherapy, levels remain the same as those seen in the pretrial era for women with tumors with scores of $\geq 31$, or that women with tumors with scores of 0-25 continue to use chemotherapy at pretrial levels seen among women with tumors with scores 0-10. 3) Score category assignment: Because score categories were based on regression results to impute scores for women who were not tested, we varied the score category assignments by the upper or lower $95 \%$ CI such that overall assignment probability remained constant at 100\%. Thus, if one category was assumed to be at the upper $95 \%$ CI, then other categories were adjusted downwards. $\mathrm{CI}=$ confidence interval. 
Table 4. Total initial care costs and savings from pre- to posttrial under differing scenarios and alternative assumptions*

2018 Costs, in Millions (US\$)

\begin{tabular}{|c|c|c|c|c|c|c|c|c|c|c|}
\hline \multirow[b]{3}{*}{ Scenario } & & & \multicolumn{4}{|c|}{$\begin{array}{c}\text { SEER chemotherapy underreporting } \\
\text { assumption }\end{array}$} & \multicolumn{4}{|c|}{$\begin{array}{l}\text { Higher cost assumption for women } \\
\text { ages } 15-64 \text { years }\end{array}$} \\
\hline & \multicolumn{2}{|c|}{ Base analysis } & \multicolumn{2}{|c|}{$7 \%$} & \multicolumn{2}{|c|}{$21 \%$} & \multicolumn{2}{|c|}{1.2} & \multicolumn{2}{|c|}{1.4} \\
\hline & Savings & $\begin{array}{l}\text { Total } \\
\text { costs }\end{array}$ & Savings & $\begin{array}{l}\text { Total } \\
\text { costs }\end{array}$ & Savings & $\begin{array}{l}\text { Total } \\
\text { costs }\end{array}$ & Savings & $\begin{array}{l}\text { Total } \\
\text { costs }\end{array}$ & Savings & $\begin{array}{l}\text { Total } \\
\text { costs }\end{array}$ \\
\hline Pretrial & - & $\$ 2.816$ & - & $\$ 2.789$ & - & $\$ 2.841$ & - & $\$ 2.676$ & - & $\$ 2.956$ \\
\hline Posttrial, 100\% compliance (base) & $\$ 49$ & $\$ 2.766$ & $\$ 22$ & $\$ 2.766$ & $\$ 74$ & $\$ 2.766$ & $\$ 36$ & $\$ 2.639$ & $\$ 62$ & $\$ 2.893$ \\
\hline Score $16-25$, age $<50,100 \%$ chemo use & $-\$ 105$ & $\$ 2.920$ & $-\$ 132$ & $\$ 2.920$ & $-\$ 80$ & $\$ 2.920$ & $-\$ 106$ & $\$ 2.782$ & $-\$ 104$ & $\$ 3.059$ \\
\hline Score $\geq 31$ pretrial chemo use & $\$ 77$ & $\$ 2.738$ & $\$ 58$ & $\$ 2.730$ & $\$ 95$ & $\$ 2.746$ & $\$ 63$ & $\$ 2.613$ & $\$ 91$ & $\$ 2.864$ \\
\hline Score $26-30$ pretrial chemo use & $\$ 75$ & $\$ 2.410$ & $\$ 54$ & $\$ 2.734$ & $\$ 93$ & $\$ 2.747$ & $\$ 61$ & $\$ 2.615$ & $\$ 88$ & $\$ 2.867$ \\
\hline Score $\geq 26$ pretrial chemo use & $\$ 102$ & $\$ 2.713$ & $\$ 90$ & $\$ 2.699$ & $\$ 114$ & $\$ 2.727$ & $\$ 88$ & $\$ 2.588$ & $\$ 117$ & $\$ 2.838$ \\
\hline Score $0-25$ pretrial chemo use & $\$ 0$ & $\$ 2.816$ & $-\$ 25$ & $\$ 2.814$ & $\$ 23$ & $\$ 2.817$ & $-\$ 11$ & $\$ 2.687$ & $\$ 11$ & $\$ 2.945$ \\
\hline Score 0-10: Lower 95\% CI & $\$ 44$ & $\$ 2.771$ & $\$ 17$ & $\$ 2.771$ & $\$ 69$ & $\$ 2.771$ & $\$ 32$ & $\$ 2.644$ & $\$ 57$ & $\$ 2.899$ \\
\hline Score 0-10: Upper 95\% CI & $\$ 55$ & $\$ 2.761$ & $\$ 27$ & $\$ 2.761$ & $\$ 79$ & $\$ 2.761$ & $\$ 41$ & $\$ 2.634$ & $\$ 68$ & $\$ 2.888$ \\
\hline Score 11-15: Lower 95\% CI & $\$ 44$ & $\$ 2.772$ & $\$ 16$ & $\$ 2.772$ & $\$ 68$ & $\$ 2,772$ & $\$ 31$ & $\$ 2.645$ & $\$ 56$ & $\$ 2.899$ \\
\hline Score 11-15: Upper 95\% CI & $\$ 55$ & $\$ 2.760$ & $\$ 28$ & $\$ 2.760$ & $\$ 80$ & $\$ 2.760$ & $\$ 42$ & $\$ 2.634$ & $\$ 68$ & $\$ 2.887$ \\
\hline Score 16-25: Lower 95\% CI & $\$ 40$ & $\$ 2.776$ & $\$ 13$ & $\$ 2.776$ & $\$ 65$ & $\$ 2.776$ & $\$ 27$ & $\$ 2.648$ & $\$ 52$ & $\$ 2.904$ \\
\hline Score 16-25: Upper 95\% CI & $\$ 59$ & $\$ 2.757$ & $\$ 32$ & $\$ 2.757$ & $\$ 84$ & $\$ 2.757$ & $\$ 46$ & $\$ 2.630$ & $\$ 73$ & $\$ 2.883$ \\
\hline Score 26-30: Lower $95 \%$ CI & $\$ 67$ & $\$ 2.749$ & $\$ 40$ & $\$ 2.749$ & $\$ 92$ & $\$ 2.749$ & $\$ 53$ & $\$ 2.623$ & $\$ 81$ & $\$ 2.875$ \\
\hline Score 26-30: Upper 95\% CI & $\$ 32$ & $\$ 2.784$ & $\$ 5$ & $\$ 2.784$ & $\$ 57$ & $\$ 2.784$ & $\$ 20$ & $\$ 2.656$ & $\$ 44$ & $\$ 2.912$ \\
\hline Score $\geq 31$ : Lower $95 \%$ CI & $\$ 66$ & $\$ 2.750$ & $\$ 39$ & $\$ 2.750$ & $\$ 91$ & $\$ 2.750$ & $\$ 52$ & $\$ 2.624$ & $\$ 79$ & $\$ 2.876$ \\
\hline Score $\geq 31$ : Upper $95 \%$ CI & $\$ 33$ & $\$ 2.783$ & $\$ 6$ & $\$ 2.783$ & $\$ 58$ & $\$ 2.783$ & $\$ 21$ & $\$ 2.655$ & $\$ 45$ & $\$ 2.911$ \\
\hline
\end{tabular}

*Among women ages 15-74 years with node-negative, hormone receptor-positive, HER2-negative, and sizes $1.1-5.0 \mathrm{~cm}$ breast cancers. Initial care costs are the total cancer costs in the first 12 months after diagnosis. Savings are the difference in total costs for a given scenario and the pretrial total costs. A negative number indicates cost increases. Pretrial data were based on observed rates of use of Oncotype DX testing in the SEER data linked to Oncotype DX data from Genomic Health Inc for women diagnosed in 2010-2015. Chemotherapy use was estimated using SEER and SEER-Medicare data. Posttrial cost scenario (base) assumed 100\% use of Oncotype testing and combined observed with predicted score distributions from pretrial data using categorical logistic regression estimates for untested women (see Supplementary Table 2, available online). Chemotherapy rates assumed compliance with trial results (ie, $0 \%$ chemotherapy among women whose tumors had an Oncotype DX recurrence score of $0-25$, and $100 \%$ chemotherapy with scores of $\geq 26$ ). Other scenarios included different adherence to score-suggested treatment and Oncotype DX score category assignment (Supplementary Table 3, available online). All one-way sensitivity analyses were repeated separately while varying correction factors for the estimation of chemotherapy rate of $7 \%$ and $21 \%$ (vs $14 \%$ assumed in base analysis) and a cost inflation adjustment of 1.2 and 1.4 among (vs 1.3 assumed in base analysis) both for women 64 years or younger (see Supplementary Tables 5 and 6, available online). CI = confidence interval; SEER = Surveillance, Epidemiology, and End Results.

and risk of recurrence (3), Oncotype DX scores have imperfect sensitivity and specificity (3). Hence, some women with scores from 11 to 25 could forego chemotherapy but may actually be destined to have a recurrence. Consequently, the ultimate costs and cost-effectiveness of the expected shift in care following the TAILORx trial will depend on the accuracy of tumor genomic test results in predicting distant recurrences and the costs associated with false-negative or false-positive scores in predicting recurrences. Past economic analyses of the lifetime impact of selecting treatment based on Oncotype DX scores suggest that with $100 \%$ testing and $100 \%$ score-suggested therapy, testing would be considered cost-effective by current standards (18). However, over the entire lifetime costs of cancer care, scoresuggested care would not be cost saving because of test misclassification and recurrences that occur despite chemotherapy.

Fourth, because the distribution of RS across all tumors in the population was not known, we used data on the joint relationships of known characteristics and RS among tested women with breast cancer to estimate scores for the whole breast cancer population, assuming the same effects of the characteristics on scores among untested and tested women. Although we tested the impact on costs of varying the range of women in each score category group, there are no data to confirm or refute our underlying assumption, but similar results were obtained when other multiple imputation methods were utilized (31) (data not shown).

Fifth, we used data on older women and available comparisons with women ages 15-64 years to estimate costs and chemotherapy use among the latter group. The resulting estimates of chemotherapy use for women younger than 65 years were similar to those reported in other studies (7), including a recent study (17) that reported initial costs for stage I breast cancer, a group similar to our study population. Our adjustment was also consistent with prior studies that showed care to be more intensive for younger women with breast cancer in different health-care settings $(32,33)$. We did not include women aged 75 years and older as they were not included in the TAILORx trial. However, some of these older women with breast cancer will be in good health and might have Oncotype DX testing to guide treatment decisions.

Sixth, we focused on costs associated with Oncotype DX because this was the test used in the TAILORx trial and is the most common gene-expression profile test in the United States. However, there are other tests available. It will be important to reassess costs once there is evidence on treatment effects using these other tests. Additionally, we did not include hormonal therapy costs because we assumed that the TAILORx trial results would not change use of this modality. Finally, we did not consider changes in the costs of Oncotype DX testing. If Oncotype DX costs were to decrease, then there would be an increase in the savings associated with personalized care among women with early-stage breast cancers based on the results of the TAILORx trial.

In summary, practice changes based on evidence from the TAILORx trial on using tumor genomic profiles to personalize 
care could result in small decreases in US national cancer care costs in the initial 12 months post-breast cancer diagnosis. Longer-term studies will be needed to evaluate the true longterm economic impact and nonmonetary benefits of personalized breast cancer care.

\section{Funding}

This work was supported in part by the National Institutes of Health under National Cancer Institute (NCI) Grant U01CA12958. The research was also supported in part from Grant R35 CA197289 to JM and supplemental funding provided by NCI's Coordinating Center for Clinical Trials and a Lombardi Comprehensive Cancer Center American Cancer Society Young Investigator Award (ACS IRG 92-152-20) and the Cancer Prevention Research Fellowship sponsored by the American Society of Preventive Oncology and Breast Cancer Research Foundation (ASPO-17-001) to JJ.

\section{Notes}

Affiliations of authors: Division of Cancer Control and Population Sciences at the National Cancer Institute, National Institutes of Health, Rockville, MD (AM, VP, LE, KJH, EJF); Department of Oncology, Georgetown University Medical Center and Cancer Prevention and Control Program, Georgetown-Lombardi Comprehensive Cancer Center, Washington, DC, (JJ, JSM); Departments of Family and Social Medicine and Epidemiology and Population Health, Albert Einstein College of Medicine, Bronx, NY (CBS).

The authors are responsible for the research and had full independence in designing the study, interpreting the data, writing, and publishing the report. The content is solely the responsibility of the authors and does not represent the official views of the National Institutes of Health. The authors have no relationships or conflicts of interest to disclose relevant to this study.

Author contributions: Angela Mariotto, Eric Feuer, Kathy J. Helzlsouer, and Jeanne Mandelblatt were responsible for conception, design, data analyses, interpretation of results, manuscript preparation, and approval of the final manuscript. Jinani Jayasekera, Valentina Petvok, Lindsey Enewold, and Clyde Schechter were responsible for design, data analyses, interpretation of results, manuscript preparation, and approval of the final manuscript.

\section{References}

1. Sparano JA, Gray RJ, Makower DF, et al. Adjuvant chemotherapy guided by a 21-gene expression assay in breast cancer. N Engl J Med. 2018;379(2):111-121.

2. Sparano JA, Gray RJ, Makower DF, et al. Prospective validation of a 21-gene expression assay in breast cancer. N Engl J Med. 2015;373(21):2005-2014.

3. Paik S, Shak S, Tang G, et al. A multigene assay to predict recurrence of tamoxifen-treated, node-negative breast cancer. N Engl J Med. 2004;351(27): 2817-2826.

4. Howlader N, Cronin KA, Kurian AW, Andridge R. Differences in breast cancer survival by molecular subtypes in the United States. Cancer Epidemiol Biomarkers Prev. 2018;27(6):619-626.

5. Harris LN, Ismaila N, McShane LM, et al. Use of biomarkers to guide decisions on adjuvant systemic therapy for women with early-stage invasive breast cancer: American Society of Clinical Oncology clinical practice guideline. J Clin Onc. 2016;34(10):1134+

6. National Comprehensive Cancer Network (2017). NCCN Clinical Practice Guidelines in Oncology. Breast Cancer. Version 2. 2017. http://www.nccn.org/ professionals/physician_gls/pdf/breast.pdf. Accessed May 30, 2017

7. Potosky AL, O'Neill SC, Isaacs C, et al. Population-based study of the effect of gene expression profiling on adjuvant chemotherapy use in breast cancer patients under the age of 65 years. Cancer. 2015;121(22):4062-4070.
8. Ray GT, Mandelblatt J, Habel LA, et al. Breast cancer multigene testing trends and impact on chemotherapy use. Am J Manag Care. 2016;22(5):E153-E160.

9. Lieu TA, Ray GT, Prausnitz SR, et al. Oncologist and organizational factors associated with variation in breast cancer multigene testing. Breast Cancer Res Treat. 2017;163(1):167-176.

10. Surveillance, Epidemiology, and End-Results (SEER) program. https://seer. cancer.gov/about/. Accessed September 2018.

11. US Census Bureau. 2017 National Population Projections Dataset. https:// www.census.gov/data/datasets/2017/demo/popproj/2017-popproj.html. Accessed September 2018

12. Petkov VI, Miller DP, Howlader N, et al. Breast-cancer-specific mortality in patients treated based on the 21-gene assay: a SEER population-based study. NPJ Breast Cancer. 2016;2:16017. doi: 10.1038/npjbcancer.2016.17.

13. Noone AM, Lund JL, Mariotto A, et al. Comparison of SEER treatment data with Medicare claims. Med Care. 2016;54(9):e55-e64.

14. Warren JL, Klabunde CN, Schrag D, Bach PB, Riley GF. Overview of the SEERMedicare data: content, research applications, and generalizability to the United States elderly population. Med Care. 2002;40(suppl 8): IV-3-IV-18.

15. Mariotto AB, Yabroff KR, Shao YW, Feuer EJ, Brown ML. Projections of the cost of cancer care in the United States: 2010-2020. J Natl Cancer Inst. 2011;103(2): 117-128.

16. Yabroff KR, Lamont EB, Mariotto A, et al. Cost of care for elderly cancer patients in the United States. J Natl Cancer Inst. 2008;100(9):630-641.

17. Banegas MP, Yabroff KR, O'Keeffe-Rosetti MC, et al. Medical care costs associated with cancer in integrated delivery systems. J Natl Compr Canc Netw. 2018, 16(4):402-410.

18. Chandler Y, Schechter CB, Jayasekera J, et al. Cost effectiveness of gene expression profile testing in community practice. J Clin Oncol. 2018;36(6): 554-562.

19. US Department of Labor, Bureau of Labor Statistics. Measuring Price Change for Medical Care in the CPI. Washington, DC: BLS; 2010. https://www.bls.gov/opub/ ted/2019/consumer-price-index-2018-in-review.htm.

20. Joh JE, Esposito NN, Kiluk JV, et al. The effect of oncotype dx recurrence score on treatment recommendations for patients with estrogen receptor-positive early stage breast cancer and correlation with estimation of recurrence risk by breast cancer specialists. Oncologist. 2011;16(11):1520-1526.

21. Sulayman N, Spellman E, Graves KD, et al. Psychosocial and quality of life in women receiving the 21-gene recurrence score assay: the impact of decision style in women with intermediate RS. J Canc Epidemiol. 2012;2012:728290. doi: 10.1155/2012/728290.

22. Hawley ST, Janz NK, Griffith KA, et al. Recurrence risk perception and quality of life following treatment of breast cancer. Breast Cancer Res Treat. 2017; 161(3):557-565.

23. Panattoni L, Lieu TA, Jayasekera J, et al. The impact of gene expression profile testing on confidence in chemotherapy decisions and prognostic expectations. Breast Cancer Res Treat. 2018;173(2):417-427.

24. Lea DH, Kaphingst KA, Bowen D, Lipkus I, Hadley DW. Communicating genetic and genomic information: health literacy and numeracy considerations. Public Health Genom. 2011;14(4-5):279-289.

25. Lo SS, Mumby PB, Norton J, et al. Prospective multicenter study of the impact of the 21-gene recurrence score assay on medical oncologist and patient adjuvant breast cancer treatment selection. J Clin Onc. 2010;28(10): 1671-1676.

26. Friese CR, Li Y, Bondarenko I, et al. Chemotherapy decisions and patient experience with the recurrence score assay for early-stage breast cancer. Cancer. 2017;123(1):43-51.

27. Ademuyiwa FO, Miller A, O'Connor T, et al. The effects of oncotype DX recurrence scores on chemotherapy utilization in a multi-institutional breast cancer cohort. Breast Cancer Res Treat. 2011;126(3):797-802.

28. Patel M, Patel K. The oncology care model: aligning financial incentives to improve outcomes. Oncol Pract Manag. 2016;6(12). http://oncpracticemanagement.com/issue-archive/2016/december-2016-vol-6-no-12/the-oncology-caremodel-aligning-financial-incentives-to-improve-outcomes/

29. Kline R, Adelson K, Kirshner JJ, et al. The oncology care model: perspectives from the Centers for Medicare \& Medicaid Services and participating oncology practices in academia and the community. Am Soc Clin Oncol Educ Book. 2017;37(37):460-466.

30. Bach PB. Costs of cancer care: a view from the centers for Medicare and Medicaid Services. J Clin Oncol. 2007;25(2):187-190.

31. Jayasekera J, Schechter CB, Sparano JA, et al. Effects of radiotherapy in earlystage, low-recurrence risk, hormone-sensitive breast cancer. J Natl Canc Inst. 2018;110(12):1370-1379.

32. Merrill RM, Brown ML, Potosky AL, et al. Survival and treatment for colorectal cancer Medicare patients in two group/staff health maintenance organizations and the fee-for-service setting. Med Care Res Rev. 1999;56(2): 177-196.

33. Smith TJ, Penberthy L, Desch CE, et al. Differences in initial treatment patterns and outcomes of lung cancer in the elderly. Lung Cancer. 1995;13(3): 235-252. 